\title{
Effects of double-layer polarization and electroosmotic flow on the electrophoresis of a finite cylinder along the axis of a cylindrical pore
}

\author{
Jyh-Ping $\mathrm{Hsu}^{\mathrm{a}, *}$, Zheng-Syun Chen ${ }^{\mathrm{a}}$, Duu-Jong Lee ${ }^{\mathrm{a}}$, Shiojenn Tseng ${ }^{\mathrm{b}}, \mathrm{Ay} \mathrm{Su}^{\mathrm{c}}$ \\ ${ }^{a}$ Department of Chemical Engineering, National Taiwan University, Taipei, Taiwan 10617, Taiwan \\ ${ }^{\mathrm{b}}$ Department of Mathematics, Tamkang University, Tamsui, Taipei, Taiwan 25137, Taiwan

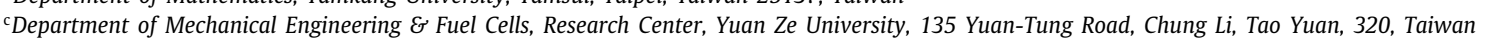

\section{A R T I C L E I N F O}

\section{Article history:}

Received 3 February 2008

Received in revised form 25 June 2008

Accepted 27 June 2008

Available online 2 July 2008

\section{Keywords:}

Colloidal phenomena

Electroosmosis

Electrophoresis

Mathematical modeling

Boundary effect

Double-layer polarization

\begin{abstract}
A B S T R A C T
The electrophoresis of a rigid, finite cylindrical particle along the axis of a long cylindrical pore is analyzed theoretically under the conditions of arbitrary surface potential and double-layer thickness. The effects of double-layer polarization and electroosmotic flow on the electrophoretic behavior of the particle are discussed. We show that if both the particle and the pore are positively charged, the mobility of the particle has a local minimum as the thickness of double layer varies. Also, if the level of the surface potential of the particle is sufficiently high and its aspect ratio is sufficiently large, then its mobility may change its sign twice as the thickness of double layer (or the concentration of electrolytes) varies. These findings are of practical significance in designing an electrophoresis operation because it will influence the prediction of the charged conditions on a particle if electrophoresis is used as an analytical tool, and the separation efficiency, if it is adopted as a separation technique.
\end{abstract}

(c) 2008 Elsevier Ltd. All rights reserved.

\section{Introduction}

Electrophoresis, the movement of a charged entity in an electrolyte solution as a response to an applied electric field, is one of the most important electrokinetic phenomena. The earliest reported theoretic analysis on electrophoresis was conducted by Helmholtz (1879). Because the dielectric effect of the liquid medium was neglected in his analysis, the applications of the result obtained are limited. The analysis of Helmholtz was extended by Von Smoluchowski (1918) to take that effect into account. Assuming infinitely thin double layer, low surface potential, and steady state, he was able to derive, for a rigid sphere in an infinite medium, $\mu=v / E=\varepsilon \zeta / \eta$, with $\mu, v, \zeta, E, \varepsilon$, and $\eta$ being the mobility, the velocity, and the surface (zeta) potential of the entity, the strength of the applied electric field, and the dielectric constant and the viscosity of the liquid phase, respectively. The corresponding result for the case of infinitely thick double layer is two-thirds that of Smoluchowski, and in general, for an arbitrarily thick double layer (Henry, 1931),

$\mu=\frac{\varepsilon \varsigma}{\eta} f(\kappa a)$

\footnotetext{
* Corresponding author. Tel.: +8862 23637448; fax: +886223623040 E-mail address: jphsu@ntu.edu.tw (J.-P. Hsu).
}

where the dependence of the mobility is incorporated into the function $f(\kappa a),(1 / \kappa)$ and $a$ being the reciprocal Debye length and the particle radius, respectively. Subsequent studies that considered more general case, such as non-spherical particles, arbitrary level of surface potentials, arbitrary thick double layers, and the presence of neighboring particles, are ample in the literature. In general, because the equations governing electrophoresis are coupled and highly nonlinear, solving them analytically is almost impossible, and a numerical approach is usually adopted (e.g., Wiersema et al., 1966; O'Brien and White, 1978). Alternatively, the governing equations are solved by assuming conditions regarding the level of surface potential and the thickness of double layer (e.g., Overbeek, 1943; Dukhin and Semenikhin, 1970; Ohshima et al., 1983). In addition to the factors stated above, the presence of a boundary adds another degree of difficulty to the solution procedure of an electrophoresis problem because the concentration, flow, and electric fields surrounding a particle will all be influenced as it approaches a boundary. In particular, if the boundary is charged, then electroosmotic flow is present, which will also affect the movement of the particle.

The presence of boundary effect is common in many applications of electrophoresis. Among the possible geometries that are of practical significance a particle in a cylindrical pore has been considered by many investigators. Often, a spherical particle is assumed, for simplicity. However, because non-spherical particles are encountered frequently, extending relevant analyses to these types of particles is highly desirable. In particular, many inorganic and biological 
entities are better described by a cylindrical particle. Typical example includes clay, protein, and DNA. Previous attempts made on the modeling of the electrophoresis of a cylindrical particle when boundary effect can be significant include that of Ye et al. (2002) where a circular cylindrical particle translates along the axis of a cylindrical microchannel under the conditions of thin double layer and low surface potential. Assuming low surface potential and weak applied electric field, Hsu and Kao (2002) investigated the influence of the charged conditions of a short cylinder on its electrophoresis along the axis of a cylindrical pore. Under the same conditions, Hsu and $\mathrm{Ku}$ (2005) discussed the influence of a charged cylindrical pore on the electrophoretic behavior of a finite cylinder. The analysis of Hsu and Kao (2002) was extended by Hsu and Kuo (2006) to the case of an eccentrically positioned finite cylinder translating parallel to the axis of a long cylindrical pore. Liu et al. (2004) considered the electrophoresis of a cylindrical particle in a long cylindrical pore under the conditions of large (radius of a particle/radius of a pore) ratio and low surface potential. For the case of thin double layer and low surface potential, Davison and Sharp (2006) analyzed the electrophoresis of a cylindrical particle positioned concentrically and eccentrically in a cylindrical pore. Hsieh and Keh (2007) studied the electrophoresis of a long cylindrical particle of non-uniform zeta potential distribution in the angular direction by an imposed electric field in the direction perpendicular or parallel to its axis near a plane wall for the case of a thin double layer and low surface potential.

In this study, our previous analysis on the electrophoresis of a finite cylinder along the axis of a long cylindrical pore (Hsu and $\mathrm{Ku}, 2005$ ) is extended to the case of arbitrary surface potential and double-layer thickness, taking account of the effects of double-layer polarization and electroosmotic flow. The first effect needs to be considered when the level of surface potential exceeds ca. $25 \mathrm{mV}$ and the thickness of the double layer surrounding a particle is comparable to its linear size (O'Brien and White, 1978; Lee et al., 1998). The second effect is important if a boundary is charged, and it can influence both quantitatively and qualitatively the electrophoretic behavior of a particle (Hsu and Ku, 2005; Hsu and Kuo, 2006; Liu et al., 2004). Although the incorporation of these effects into electrophoresis modeling makes the analysis much more complicated, it is highly desirable to experimentalists because experiments are usually conducted under conditions where they are significant. In our study, the influences of the thickness of the double layer, the aspect ratio of a particle, the level of surface potential, and the relative size of the pore on the electrophoretic mobility of a particle are examined.

\section{Theory}

The problem under consideration is illustrated in Fig. 1, where a rigid, non-conducting, cylindrical particle of radius $a$ and length $2 d$ translates along the axis of a long cylindrical pore of radius $b$ filled with a Newtonian fluid of constant physical properties as a response to an applied uniform electric field $\mathbf{E}$. The liquid phase contains $z_{1}$ : $z_{2}$ electrolytes; $z_{1}$ and $z_{2}$ are the valence of cations and that of anions, respectively, with $\alpha=-z_{2} / z_{1}$. Let $\mathbf{U}$ be the velocity of the particle. The cylindrical coordinates $(r, \theta, z)$ are adopted, its origin is located at the center of the particle, and $\mathbf{E}$ is in the $z$-direction. Because the present problem is $\theta$-symmetric only the $(r, z)$ domain needs to be considered.

Assuming steady state condition, the governing equations of the present problem, including those for the electrical, concentration, and flow fields, can be summarized as follows:

$\nabla^{2} \phi=-\frac{\rho}{\varepsilon}=-\sum_{j=1}^{2} \frac{z_{j} e n_{j}}{\varepsilon}$

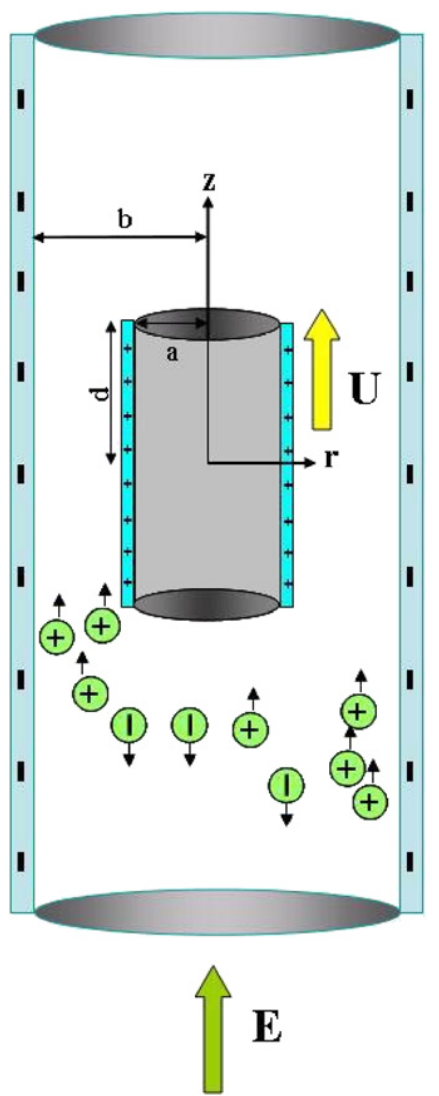

Fig. 1. The electrophoresis of a rigid cylindrical particle of radius $a$ and length $2 d$ driven by a uniform electric field $\mathbf{E}$ along the axis of a long cylindrical pore of radius $b$. The cylindrical coordinates $(r, \theta, z)$ with its origin located at the center of the particle are adopted; $\mathbf{E}$ is in the $z$-direction.

$\nabla \cdot\left[-D_{j}\left[\nabla n_{j}+\frac{z_{j} e}{k_{B} T} n_{j} \nabla \phi\right]+n_{j} \mathbf{v}\right]=0$

$\nabla \cdot \mathbf{v}=0$

$-\nabla p+\eta \nabla^{2} \mathbf{v}+\rho \nabla \phi=0$

Here, $\nabla^{2}$ is the Laplace operator, $\phi$ the electrical potential, $\varepsilon$ the permittivity of the liquid phase, $\rho$ the space charge density, $e$ the elementary charge, and $n_{j}$ and $D_{j}$ are the number concentration and the diffusivity of ionic species $j$, respectively. $k_{B}, T, \mathbf{v}, \eta$, and $p$ are the Boltzmann constant, the absolute temperature, the liquid velocity, the viscosity of the liquid, and the hydrodynamic pressure, respectively.

The mathematical treatment can be simplified by partitioning $\phi$ into the equilibrium potential, that is, the potential in the absence of $\mathbf{E}, \phi_{1}$, and the potential outside the particle when $\mathbf{E}$ is applied, $\phi_{2}, \phi=\phi_{1}+\phi_{2}$ (O'Brien and White, 1978; Lee et al., 1998). Also, the distortion of the ionic cloud surrounding the particle is expressed as

$n_{j}=n_{j 0} \exp \left(-\frac{z_{j} e\left(\phi_{1}+\phi_{2}+g_{j}\right)}{k_{B} T}\right), \quad j=1,2$

where $n_{j 0}$ and $g_{j}$ are the bulk concentration and a perturbed potential associated with ionic species $j$, respectively. Note that this does not mean that the distribution of $n_{j}$ is Boltzmann because the actual distribution depends on $g_{j}$. In practice, the upper limit of the surface potential of a particle and that of a surface is on the order of $100 \mathrm{mV}$ and the thickness of the double layer on the order of $100 \mathrm{~nm}$. This implies that the strength of the electric field established by the particle and/or the pore is on the order of $10^{3} \mathrm{kV} / \mathrm{m}$, which is much 
stronger than that of E, E. In this case, all the dependent variables can be partitioned into an equilibrium term and a perturbed term, the later is on the order of $E$. For the flow field, $\mathbf{v}$ and $p$ can expressed as $\mathbf{v}=\mathbf{v}^{(e)}+\delta \mathbf{v}$ and $p=p^{(e)}+\delta p$, respectively, where superscript ( $e$ ) and prefix $\delta$ denote the equilibrium term and the perturbed term, respectively. Substituting these expressions into Eqs. (3) and (4) and collecting terms on the order of $E$ yield the governing equations for the perturbed flow field. For convenience, the $\delta \mathbf{v}$ and $\delta p$ in these equations are replaced by $\mathbf{v}$ and $p$, respectively. Suppose that $\left(\phi_{2}+g_{j}\right) \ll\left(k_{B} T / e z_{j}\right)$. Then it can be shown that in scaled symbols the governing equations of the concentration, electrical, and flow fields for the perturbed problem are (Lee et al., 1998; Hsu et al., 2007a)

$$
\begin{aligned}
& n_{1}^{*}=\exp \left(-\phi_{r} \phi_{1}^{*}\right)\left[1-\phi_{r}\left(\phi_{2}^{*}+g_{1}^{*}\right)\right] \\
& n_{2}^{*}=\exp \left(-\phi_{r} \phi_{1}^{*}\right)\left[1-\alpha \phi_{r}\left(\phi_{2}^{*}+g_{1}^{*}\right)\right] \\
& \nabla^{* 2} \phi_{1}^{*}=-\frac{1}{(1+\alpha)} \frac{(\kappa a)^{2}}{\phi_{r}}\left[\exp \left(-\phi_{r} \phi_{1}^{*}\right)-\exp \left(\alpha \phi_{r} \phi_{1}^{*}\right)\right] \\
& \nabla^{* 2} \phi_{2}^{*}-\frac{(\kappa a)^{2}}{(1+\alpha)}\left[\exp \left(-\phi_{r} \phi_{1}^{*}\right)+\alpha \exp \left(\alpha \phi_{r} \phi_{1}^{*}\right)\right] \phi_{2}^{*} \\
& \quad=\frac{(\kappa a)^{2}}{(1+\alpha)}\left[\exp \left(-\phi_{r} \phi_{1}^{*}\right) g_{1}^{*}+\exp \left(\alpha \phi_{r} \phi_{1}^{*}\right) \alpha g_{2}^{*}\right] \\
& \nabla^{* 2} g_{1}^{*}-\phi_{r} \nabla^{*} \phi_{1}^{*} \cdot \nabla^{*} g_{1}^{*}=\phi_{r}^{2} P e_{1} \mathbf{v}^{*} \cdot \nabla^{*} \phi_{1}^{*} \\
& \nabla^{* 2} g_{2}^{*}+\alpha \phi_{r} \nabla^{*} \phi_{1}^{*} \cdot \nabla^{*} g_{2}^{*}=\phi_{r}^{2} P e_{2} \mathbf{v}^{*} \cdot \nabla^{*} \phi_{1}^{*} \\
& \nabla \cdot \mathbf{v}^{*}=0 \\
& -\nabla^{*} p-\nabla^{* 2} \mathbf{v}^{*}+\nabla^{* 2} \phi_{2}^{*} \nabla^{*} \phi_{1}^{*}+\nabla^{* 2} \phi_{1}^{*} \nabla^{*} \phi_{2}^{*}=0
\end{aligned}
$$

In these expressions, $n_{j}^{*}=n_{j} / n_{10}, \zeta_{a}$ is the surface potential of the particle, $\phi_{r}=\zeta_{a} z_{1} e / k_{B} T, \phi_{j}^{*}=\phi_{j} / \zeta_{a}, g_{j}^{*}=g_{j} / \zeta_{a}, j=1,2, \nabla^{* 2}=\nabla^{2} / a^{2}$, $\kappa=\left[\sum_{j=1}^{2} n_{j 0}\left(e z_{j}\right)^{2} / \varepsilon k_{B} T\right]^{1 / 2}$ is the reciprocal Debye length, $\nabla^{*}=\nabla / a$, $\mathbf{v}^{*}=\mathbf{v} / U_{E}, U_{E}=\left(\varepsilon \zeta_{a}^{2} / \eta a\right), P e_{j}=\varepsilon\left(z_{j} e / k_{B} T\right)^{2} / \eta D_{j}, j=1,2$, is the Peclet number of ionic species $j$, and $p^{*}=p / p_{\text {ref }}, p_{\text {ref }}=\varepsilon \xi_{a}^{2} / a^{2}$ is a reference pressure.

Suppose that both the particle and the pore are non-conductive, impermeable to ionic species, and remained at constant surface potential, the concentration of ionic species away from the particle reaches the corresponding bulk value, and both the surface of the particle and that of the pore are no-slip. Then we have the following boundary conditions:

$\phi_{1}^{*}=\zeta_{a} / \zeta_{a}$ on the particle surface

$\phi_{1}^{*}=\zeta_{b} / \zeta_{a} \quad$ on the pore surface

$\phi_{1}^{*}=\left(\zeta_{b} / \zeta_{a}\right) \frac{I_{0}(\kappa r)}{I_{0}(\kappa c)}, \quad\|z\| \rightarrow \infty, \quad r<b$

$\mathbf{n} \cdot \nabla \phi_{2}^{*}=0$ on the particle surface

$\mathbf{n} \cdot \nabla \phi_{2}^{*}=0$ on the pore surface

$\nabla \phi_{2}^{*}=-E_{z}^{*} e_{z}, \quad|z| \rightarrow \infty, \quad r<b$

n $\cdot \nabla^{*} g_{j}^{*}=0, \quad j=1,2$ on the particle surface

$\mathbf{n} \cdot \nabla^{*} g_{j}^{*}=0, \quad j=1,2$ on the pore surface

$g_{j}^{*}=-\phi_{2}^{*}, \quad j=1,2,\|z\| \rightarrow \infty, r<b$

$v^{*}=\left(v / U_{E}\right) \mathbf{e}_{z}$ on the particle surface $v^{*}=0 \quad$ on the pore surface

$v^{*}=\left(v(r) / U_{E}\right) e_{z}=-\left(\zeta_{w} / \zeta_{a}\right)\left[1-\frac{I_{0}(\kappa r)}{I_{0}(\kappa b)}\right] e_{z}$,

$\|z\| \rightarrow \infty, r<b$

In these expressions, $E_{z}^{*}=E_{z} /\left(\zeta_{a} / a\right), I_{0}$ is the zero-order modified Bessel function of the first kind, $\zeta_{b}$ is the surface potential of the pore, $v$ is the speed of the particle in the $z$-direction, and $\mathbf{e}_{z}$ is the unit vector in the $z$-direction. Note that Eq. (15) describes the undisturbed electroosmotic velocity profile, $v(r) \mathbf{e}_{z}$, for a charged cylindrical pore in the absence of the particle (Hsu and Ku, 2005).

Usually, problems of the present type need to be solved through a tedious trial-and-error procedure. This difficulty can be circumvented by partitioning the original problem into two hypothesized sub-problems (O'Brien and White, 1978). In the first sub-problem, the particle moves with a constant velocity in the absence of $\mathbf{E}$, which is a pure hydrodynamic problem. In the second sub-problem, $\mathbf{E}$ is applied but the particle is remained fixed in space. If we let $F_{1}$ and $F_{2}$ be the total forces acting on the particle in these two sub-problems, respectively, then $F_{1}=\chi U^{*}$ and $F_{2}=\beta E_{z}^{*}$, where $U^{*}=v / U_{E}$, and $\chi$ and $\beta$ are proportional constants. Therefore, we must have, at steady state,

$\omega=\frac{U^{*}}{E_{z}^{*}}=-\frac{\beta}{\chi}$

where $\omega$ is the scaled electrophoretic mobility of the particle. In our problem, the forces acting on the particle include the electrical and the hydrodynamic forces, and only the $z$-components of these forces need to be considered. Let $F_{e i}$ and $F_{d i}$ be the $z$-components of the electrical and the hydrodynamic forces in sub-problem $i$, respectively. It can be shown that (Hsu et al., 2007a,b; Hsu and Yeh, 2006; Happel and Brenner, 1983)

$F_{e i}^{*}=\frac{F_{e i}}{\varepsilon \zeta_{a}^{2}}=\int_{S} \frac{\partial \phi_{1}^{*}}{\partial n} \frac{\partial \phi_{2}^{*}}{\partial z^{*}}-\frac{\partial \phi_{1}^{*}}{\partial t} \frac{\partial \phi_{2}^{*}}{\partial t} \mathrm{~d} S^{*}$

$F_{d i}^{*}=\frac{F_{d z}}{\varepsilon \zeta_{a}^{2}}=\int_{S}\left(\sigma^{H^{*}} \cdot \mathbf{n}\right) \cdot \mathbf{e}_{z} \mathrm{~d} S^{*}$

In these expressions, $n, t$, and $z^{*}$ are the magnitude of the unit normal vector, that of the unit tangential vector, and the $z$-component of the unit normal vector, respectively. $F_{e i}^{*}$ and $F_{d i}^{*}$ are the scaled forms of $F_{e i}$ and $F_{d i}$, respectively. $S^{*}=S / a^{2}$ is the scaled particle surface, and $\sigma^{H *}$ the scaled shear stress tensor.

\section{Results and discussion}

The complicated nature of the present boundary-valued problem suggests that it needs to be solved numerically. To this end, FlexPDE (2003), a commercial software, which is based on a finite element method, is adopted. Here, we assume that the pore is sufficiently long so that the end effect of the flow field can be neglected. Our experience reveals that this can be achieved by letting $(L / d)>10, L$ being the length of the computation domain in the axial direction. Double precision is applied throughout the numerical simulation and grid independence is checked to assure that the mesh used is fine enough. Typically, using a total of 16000 and 8000 nodes is necessary for the resolution of the electric field and the flow field, respectively. A representative mesh used is shown in Fig. 2.

To justify the applicability of the software adopted, it is used to solve the problem considered by Liu et al. (2004) where the electrophoresis of a cylindrical particle in a long cylindrical pore is under the conditions of low surface potential and finite double-layer thickness was simulated; their analysis was limited to the case where the end effect of a particle is negligible $\left(L_{T} / L_{C}=100, L_{C}\right.$ and $L_{T}$ are the length of the particle and that of the pore, respectively). As seen 


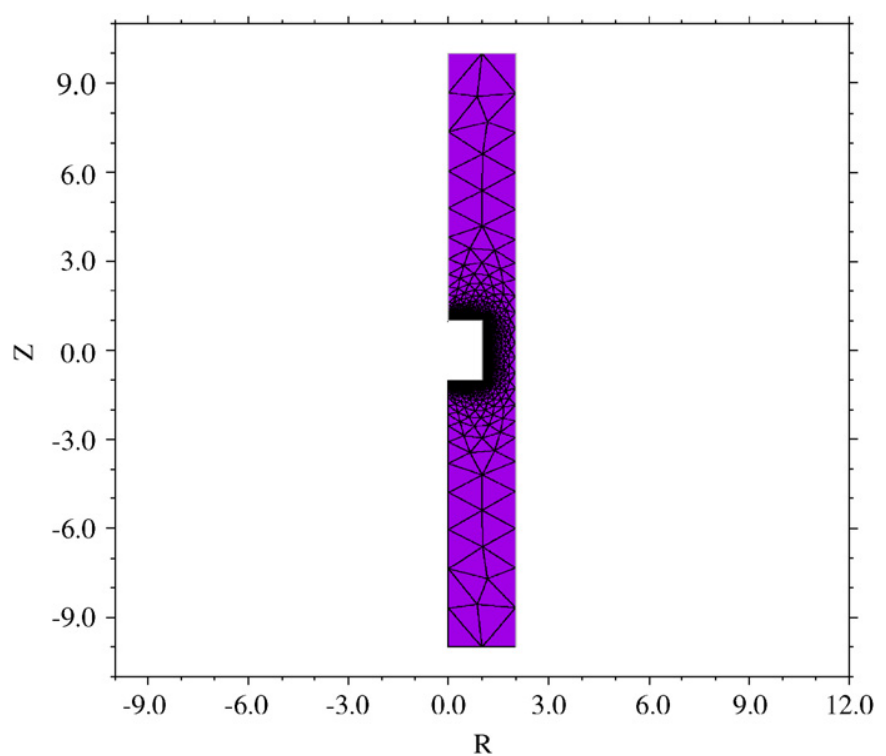

Fig. 2. Typical mesh used where the number of nodes is 8021 .

a

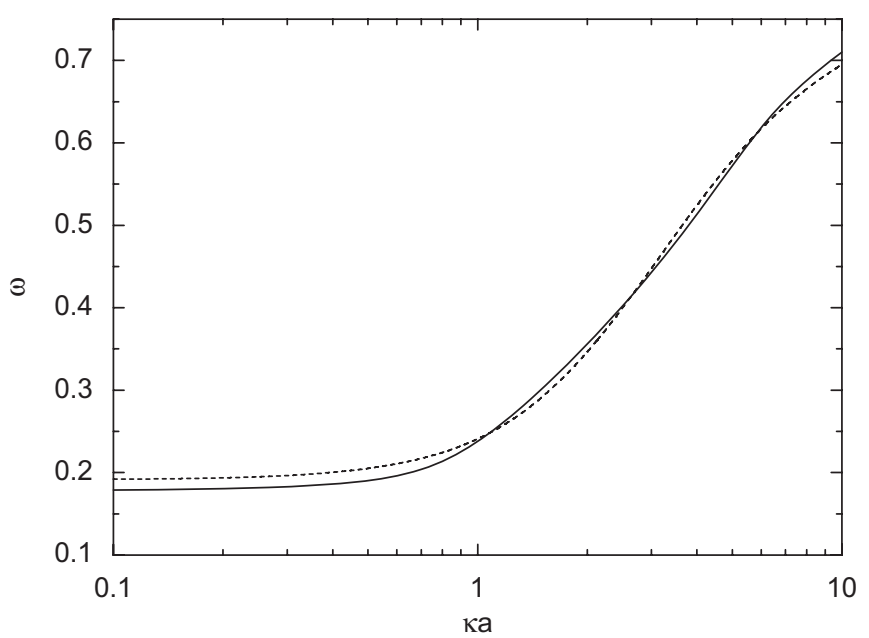

b

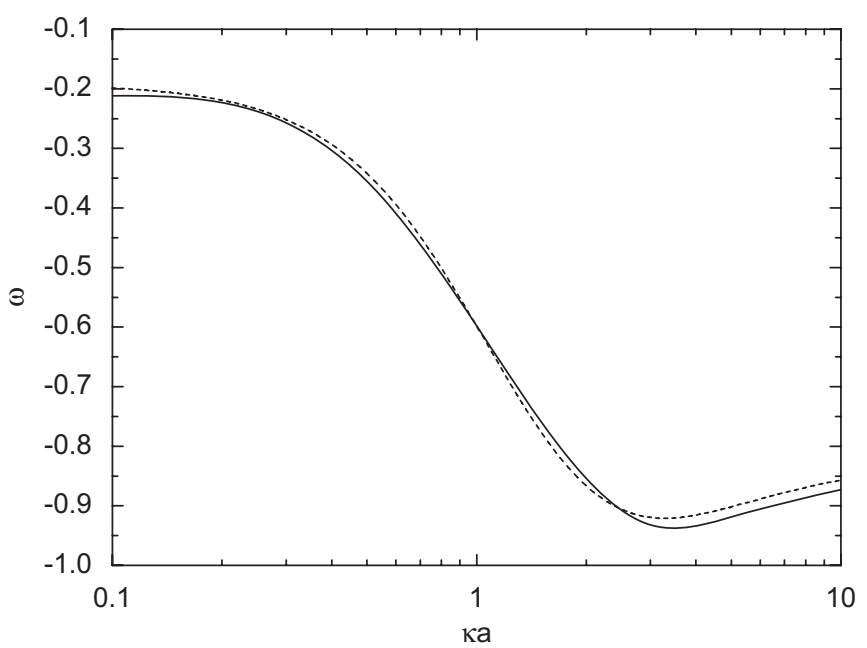

Fig. 3. Variation of the scaled electrophoretic mobility $\omega$ as a function of $\kappa a$ at $\phi_{\mathrm{r}}=1, \lambda=0.5$, and $d / a=10$. Solid curves: present numerical results; dashed curves: analytical results of Liu et al. (2004) at $L_{T} / L_{c}=100$. (a) $\zeta_{a}^{*}=1$ and $\zeta_{b}^{*}=0$, (b) $\zeta_{a}^{*}=0$ and $\zeta_{b}^{*}=1$. a

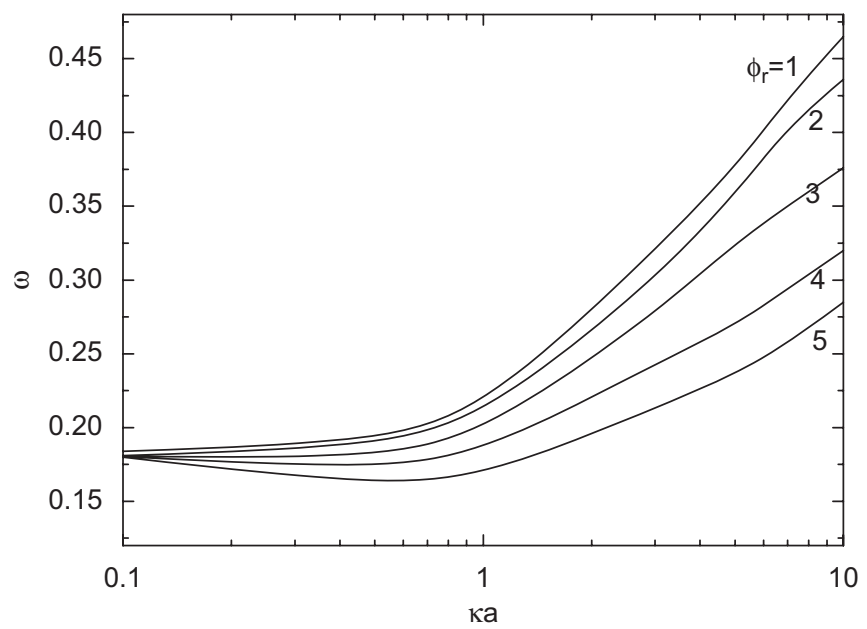

b

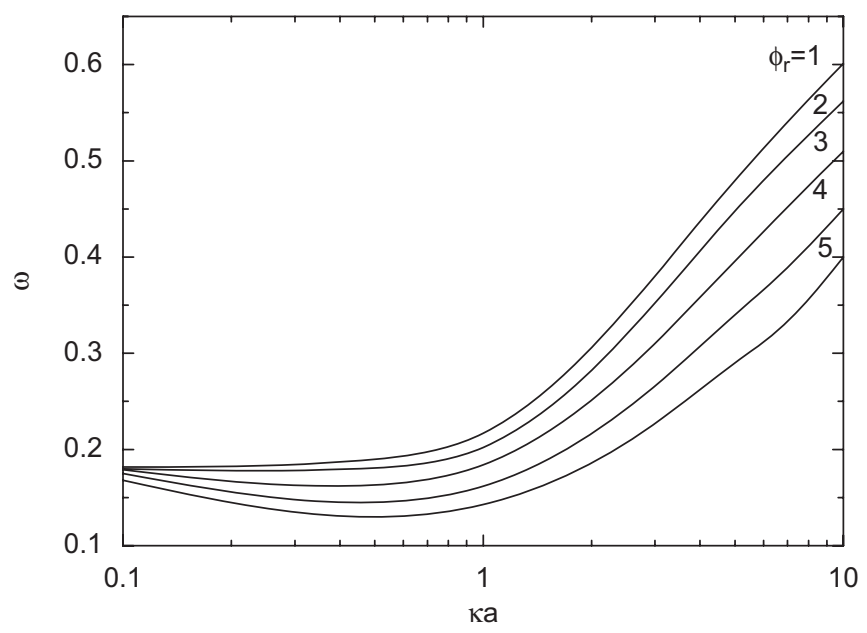

C

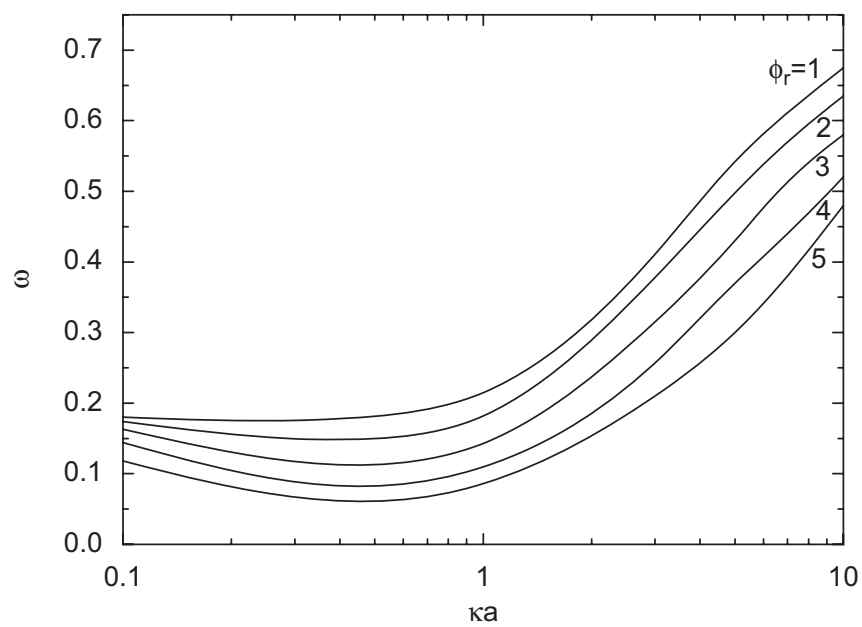

Fig. 4. Variation of the scaled electrophoretic mobility $\omega$ as a function of $\kappa a$ for various combinations of $\phi_{r}$ and $(d / a)$ at $\zeta_{b}^{*}=0$ and $\lambda=0.5$. (a) $d / a=0.25$, (b) $d / a=1$, (c) $d / a=4$.

in Fig. 3, the performance of the software adopted in our study is satisfactory, in general. Note that if the particle is charged and the pore is uncharged, the result of Liu et al. is less accurate when the double layer is thick ( $\kappa a$ small). On the other hand, if the particle is 
a

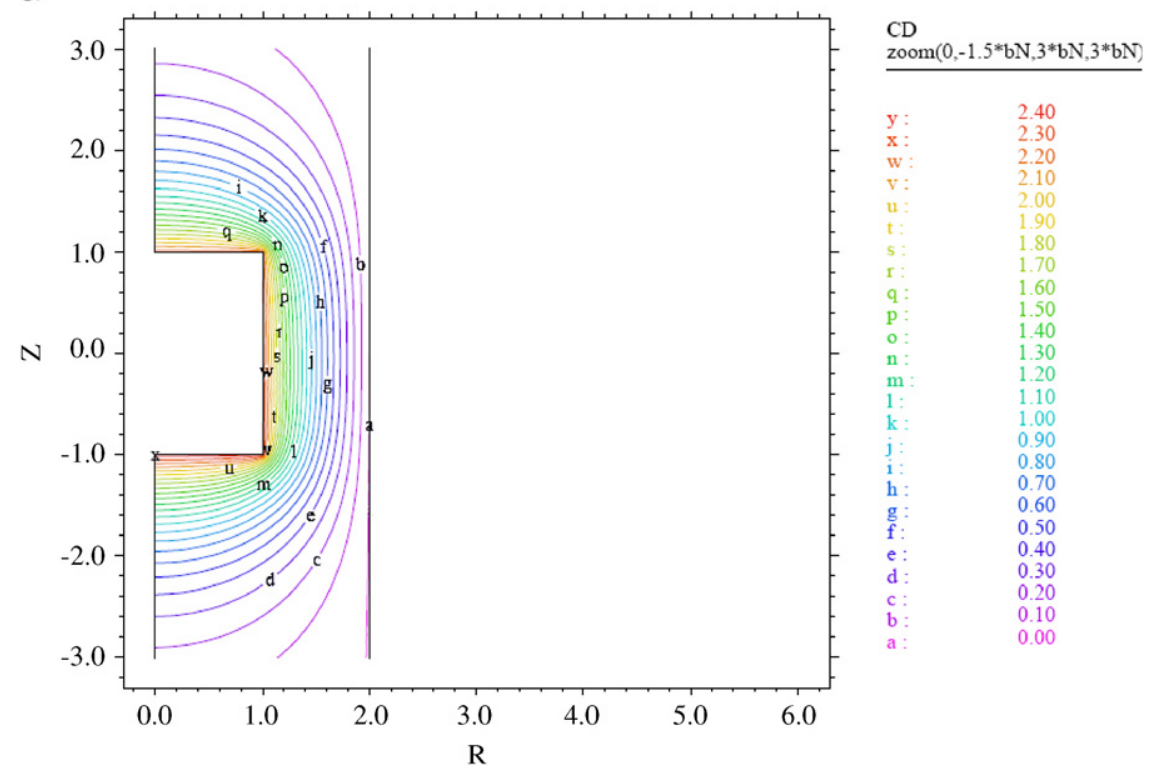

b

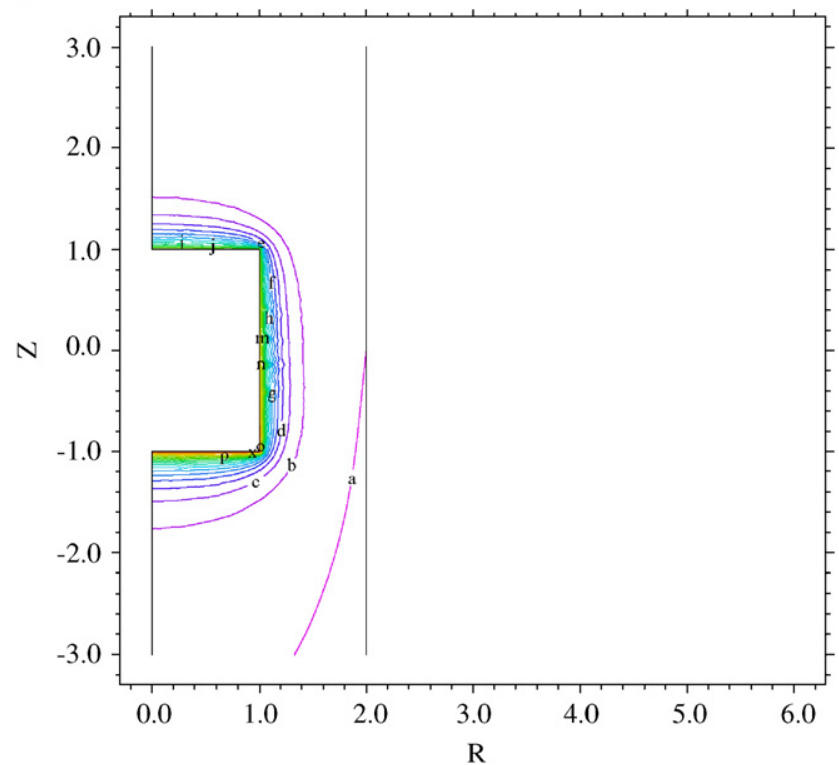

$\mathrm{CD}$ zoom $(0,-1.5 * \mathrm{bN}, 3 * \mathrm{bN}, 3 * \mathrm{bN}$

\section{0.}

180.
170.
160.

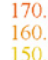

$$
\begin{aligned}
& 40.0 \\
& 30.0
\end{aligned}
$$

$$
\begin{aligned}
& 30.0 \\
& 20.0
\end{aligned}
$$$$
\begin{aligned}
& 20.0 \\
& 10.0
\end{aligned}
$$$$
0.00
$$

Fig. 5. Contours of the net scaled ionic concentration $C D\left(=n_{1}^{*}-n_{2}^{*}\right)$ for two levels of $\phi_{r}$ at $\zeta_{b}^{*}=0, \lambda=0.5, d / a=1$, and $\kappa a=0.5$. (a) $\phi_{r}=1$, (b) $\phi_{r}=5$.

uncharged and the pore is charged, their result becomes less accurate when the double layer is thin.

Let us consider two representative cases, namely, a positively charged particle in an uncharged pore, and an uncharged particle in a positively charged pore.

\subsection{Positively charged particle in an uncharged pore}

Fig. 4 illustrates the variation of the scaled electrophoretic mobility of a particle $\omega$ as a function of the thickness of the double layer, measured by $\kappa a$, at various combinations of the aspect ratio of the particle $(d / a)$ and its scaled surface potential $\phi_{r}$ for the case when a positively charged particle is placed in an uncharged pore. For illustration, the radius of the particle $a$ is fixed and the reciprocal Debye length $\kappa$ varies, that is, the concentration of electrolytes varies in the simulation. Note that for a fixed $\kappa a$, although the higher the $\phi_{r}$, the smaller the $\omega$, the actual mobility increases with $\phi_{r}$, as it should be. As seen in Fig. 4, if $\phi_{r}$ takes a small to medium large value, $\omega$ increases monotonically with the increase in $\kappa a$, but if $\phi_{r}$ is sufficiently high, $\omega$ has a local minimum as $\kappa a$ varies. The presence of the local minimum in $\omega$ as $\kappa a$ varies was also observed in the electrophoresis of an isolated sphere in an infinite fluid (Wiersema et al., 1966; O'Brien and White, 1978), and in the electrophoresis of a sphere in a cylinder pore (Hsu and Cheng, 2007). Note that for a longer particle, the local minimum occurs at a lower level of surface potential. The presence of the local minimum in $\omega$ arises from the effect of double-layer polarization, which yields an internal electric field having the direction inverse to that of the applied electric field (Lee et al., 1998; Hsu and Cheng, 2007; Hsu et al., 2007a,b). Because the strength of the induced electric field increases with the increasing $\phi_{r}$, the higher the $\phi_{r}$, the easier to observe the local minimum. The effect of double-layer polarization arises from the fact that as a particle moves in an applied electric field, the shape of the ionic cloud near itsfront region becomes asymmetric to that in its rear 
a

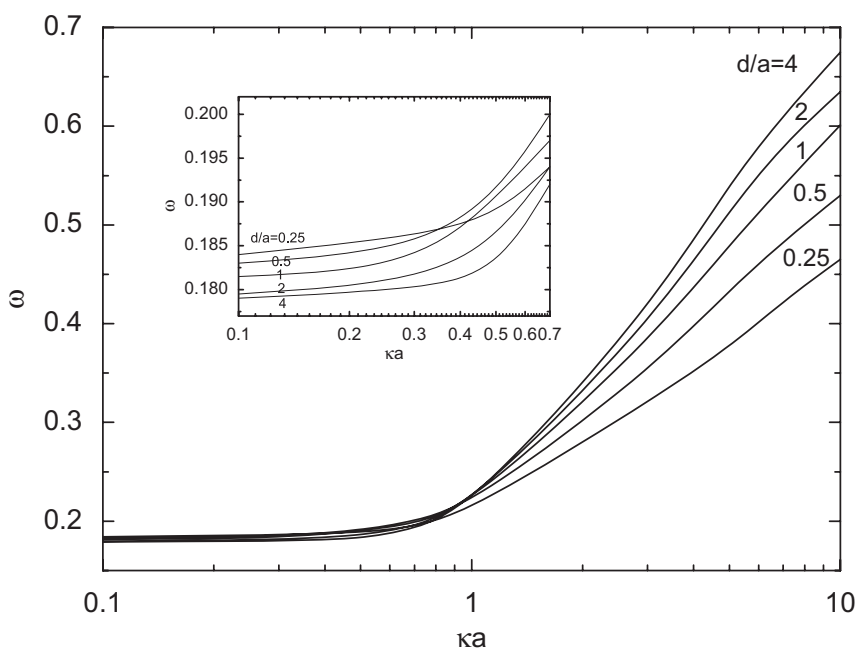

b

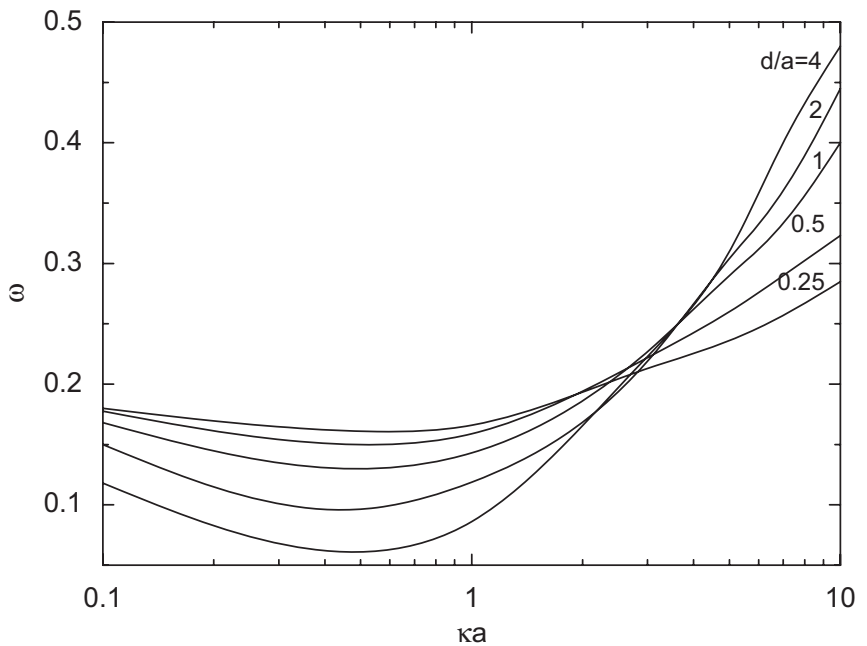

Fig. 6. Variation of the scaled electrophoretic mobility $\omega$ as a function of $\kappa a$ for various combinations of $\phi_{r}$ and $(d / a)$ at $\lambda=0.5$ and $\zeta_{b}^{*}=0$. (a) $\phi_{r}=1$, (b) $\phi_{r}=5$.

region. Fig. 5 illustrates the contours for the net scaled ionic concentration $C D\left(=n_{2}^{*}-n_{1}^{*}\right)$ at two levels of $\phi_{r}$. For the present case, because the particle is positively charged, $C D$ is positive. As seen in Fig. 5, double-layer polarization is insignificant when $\phi_{r}$ is low, but becomes appreciable if $\phi_{r}$ is sufficiently high.

The influence of the aspect ratio of a particle $(d / a)$ on its scaled mobility $\omega$ is illustrated in Fig. 6. This figure reveals that if $\kappa a$ is small, $\omega$ declines with the increase in $(d / a)$, but the reverse is true if $\kappa a$ is large. These behaviors are the consequence of the competition between the hydrodynamic drag and the electrical driving force acting on a particle as $\kappa a$ varies. For the present case, the larger the $(d / a)$, the larger the surface area of a particle, the greater the amount of surface charge, and therefore the greater the electrical driving force. The larger the surface area arising from a larger $(d / a)$ also leads to a greater hydrodynamic drag. However, if $\mathrm{ka}$ small, the rate of increase in the hydrodynamic drag as $(d / a)$ increases is faster than the rate of increase in the corresponding electrical driving force. If $\kappa a$ is large, the reverse trend is observed. Fig. 6 also reveals that if $\phi_{r}$ is sufficiently high, $\omega$ has a local minimum as $\kappa a$ varies. As mentioned previously, this arises from the effect of double-layer polarization.

Fig. 7 illustrates the boundary effect, the significance of which is measured by $\lambda(=a / b)$, on the scaled mobility of a particle $\omega$ for a

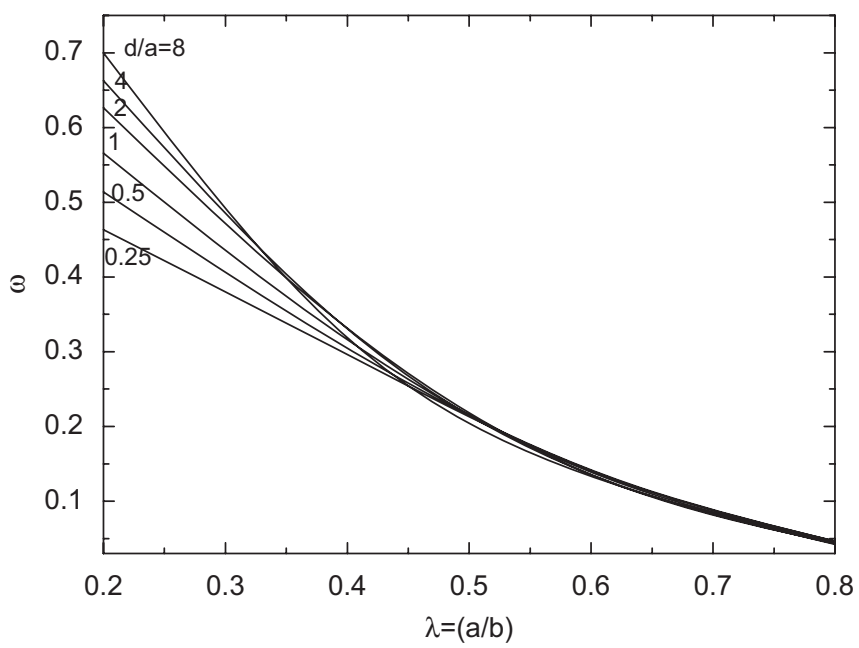

b

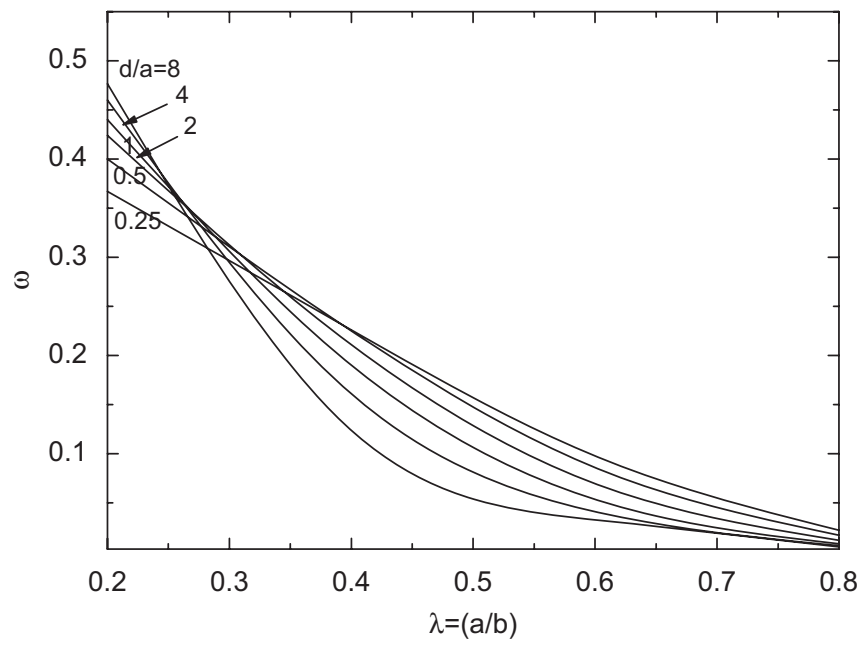

Fig. 7. Variation of the scaled electrophoretic mobility $\omega$ as a function of $\lambda(=a / b)$ for various combinations of $\phi_{r}$ and $(d / a)$ at $\zeta_{b}^{*}=0$ and $\kappa a=1$. (a) $\phi_{r}=1$, (b) $\phi_{r}=5$.

various combinations of the aspect ratio $(d / a)$ of the particle and the level of the scaled surface potential of the particle $\phi_{r}$. As seen in Fig. 7, the larger the $\lambda$, the smaller the $\omega$, that is, the presence of the pore has the effect of retarding the translation of the particle. If $\lambda$ is small, $\omega$ increases with the increasing $(d / a)$, but the reverse trend is observed if $\lambda$ is large. This can be explained by the fact that if $\lambda$ is small, the rate of increase in the electrical driving force acting on a particle as $(d / a)$ increases is faster than that of the hydrodynamic drag, but this trend becomes reversed if $\lambda$ is large. Note that for the case where $\phi_{r}$ is low, the boundary effect becomes unimportant if $\lambda$ is sufficiently large.

\subsection{Both particle and pore are positively charged}

For the present case, two additional effects that are absent in the case where a positively charged particle is placed in an uncharged pore, namely, a negative charge is induced on the particle surface as it approaches the pore, and an electroosmotic flow developed by the charged pore. Because both of these effects will reduce the electrophoretic velocity of the particle, its mobility is expected to be smaller than that of the previous case, as is justified by comparing the results presented in Figs. 8 and 4. Except that, the 
a

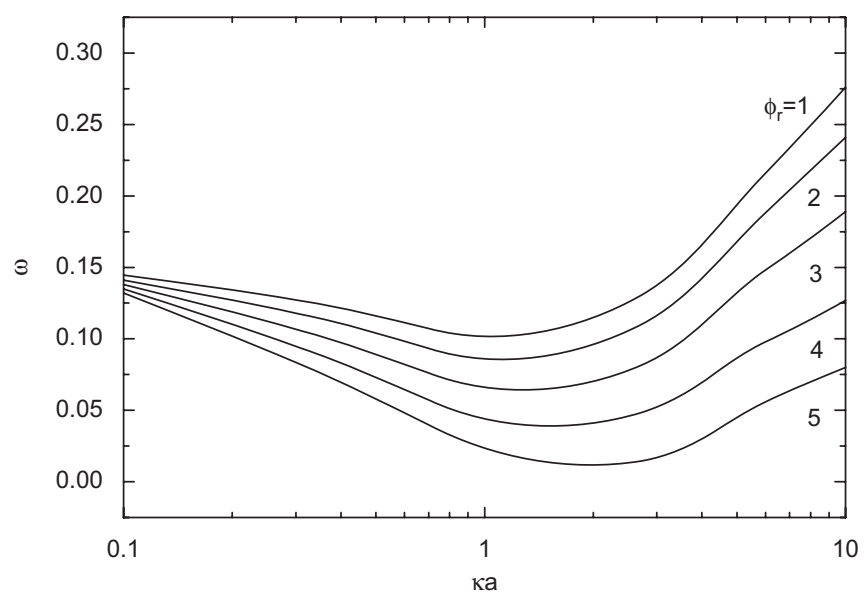

b

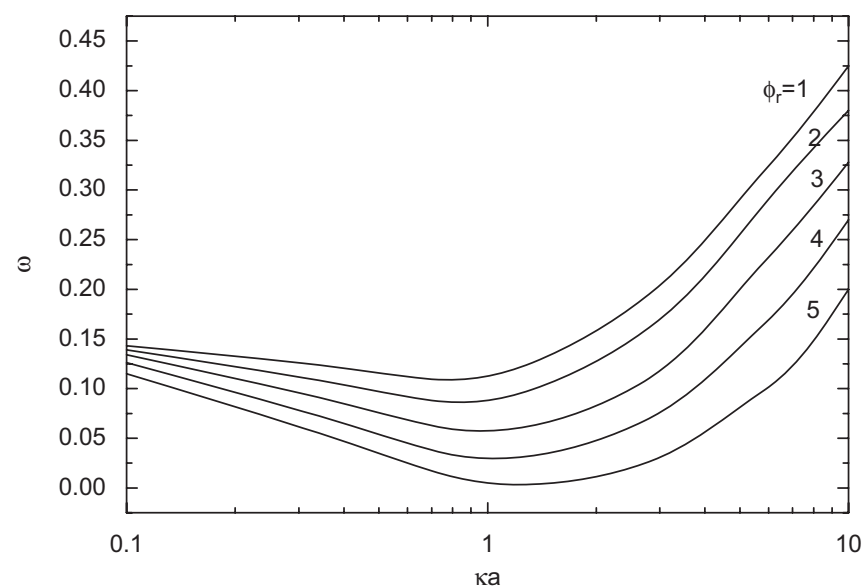

C

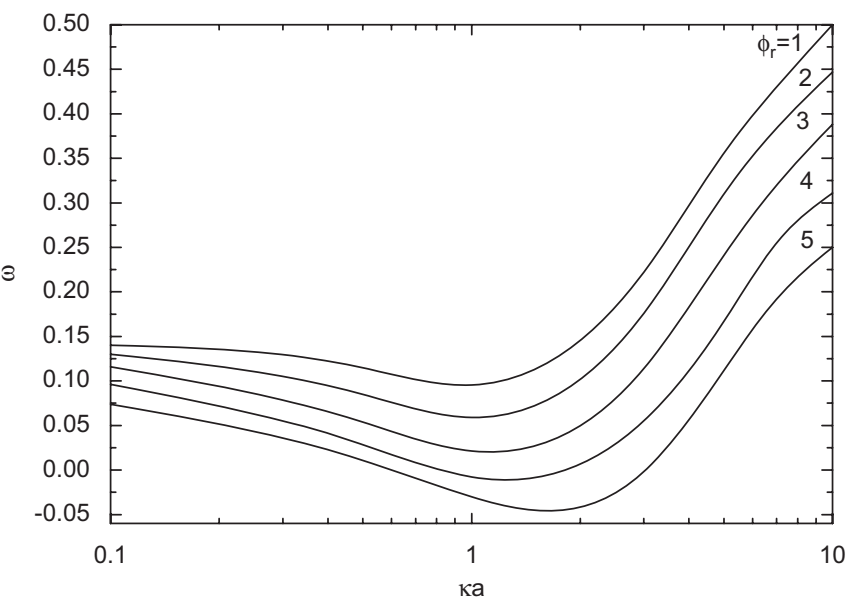

Fig. 8. Variation of the scaled electrophoretic mobility $\omega$ as a function of $\kappa a$ for various combinations of $\phi_{r}$ and $(d / a)$ at $\zeta_{b}^{*}=0.2 \zeta_{a}^{*}$ and $\lambda=0.5$. (a) $d / a=0.25$, (b) $d / a=1,($ c) $d / a=4$.

general qualitative behaviors of $\omega$ in Fig. 8 are similar to those of Fig. 4.

Fig. 9 illustrates the variation of the scaled electrophoretic mobility of a particle $\omega$ as a function of the thickness of the double layer $\kappa a$ for various aspect ratios $(d / a)$ at two levels of the scaled surface potential of the particle $\phi_{r}$. A comparison between Figs. 9 and 6 reveals that although the general behaviors of $\omega$ in these two figures a

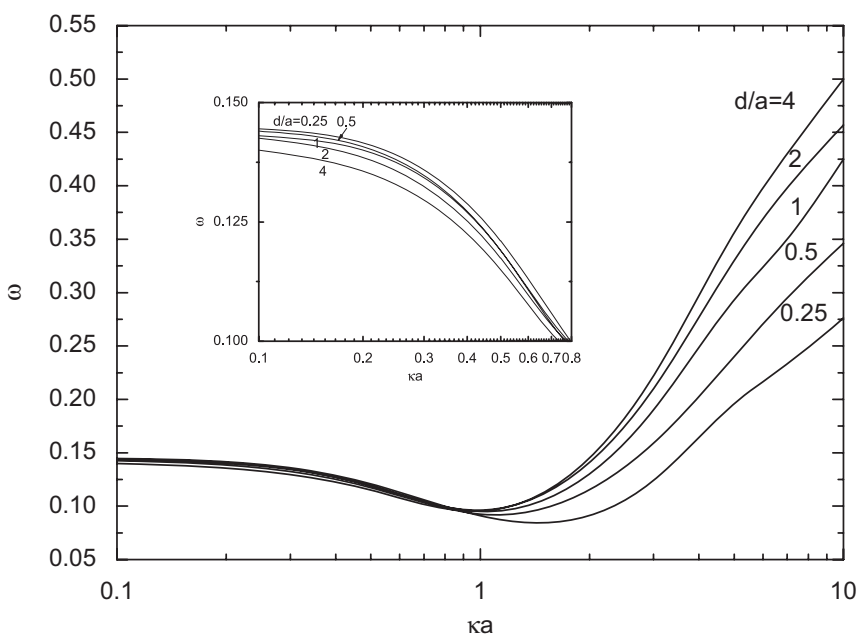

b

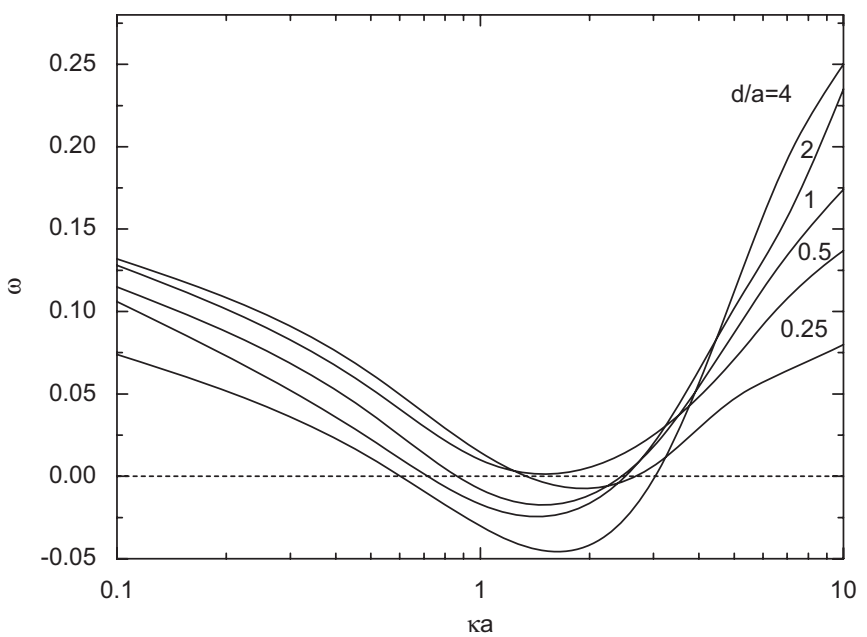

Fig. 9. Variation of the scaled electrophoretic mobility $\omega$ as a function of $\kappa a$ for various combinations of $\phi_{r}$ and $(d / a)$ at $\lambda=0.5$ and $\zeta_{b}^{*}=0.2 \zeta_{a}^{*}$. (a) $\phi_{r}=1$, (b) $\phi_{r}=5$.

seem to be similar to each other, there exist two basic differences. Firstly, if $\phi_{r}$ is low, for a small to medium large $\kappa a, \omega$ increases monotonically with the increasing $\kappa a$ in Fig. 6 , but the reverse trend is observed in Fig. 9. Secondly, while $\omega$ is always positive in Fig. 6, it may change its sign from positive to negative as $\kappa a$ increases in Fig. 9(b), where $\phi_{r}$ is high. In addition, $\omega$ may change its sign from negative to positive again if $\kappa a$ is further increased. The variations in the sign of $\omega$ as $\kappa a$ varies arises mainly from the effect of double-layer polarization, the negative charge induced on the particle surface as it approaches the positively charged pore, and the electroosmotic flow generated by the charged pore. In the present case, the polarized double layer yields a local electric field, the direction of which is opposite to that of the applied electric field. In practice, attention needs be paid to the changes in the direction of electrophoresis because it will influence the prediction of the charged conditions on a particle if electrophoresis is used as an analytical tool, or the separation efficiency if it is adopted as a separation technique. The results presented in Fig. 9 suggest that the concentration of electrolytes plays the key role in designing an electrophoresis operation.

A comparison between Figs. 10 and 7 indicates that if the scaled surface potential of a particle $\phi_{r}$ is low, the influence of the pore on the scaled electrophoretic mobility of the particle $\omega$ for the case where both the particle and the pore are positively charged is similar 
a

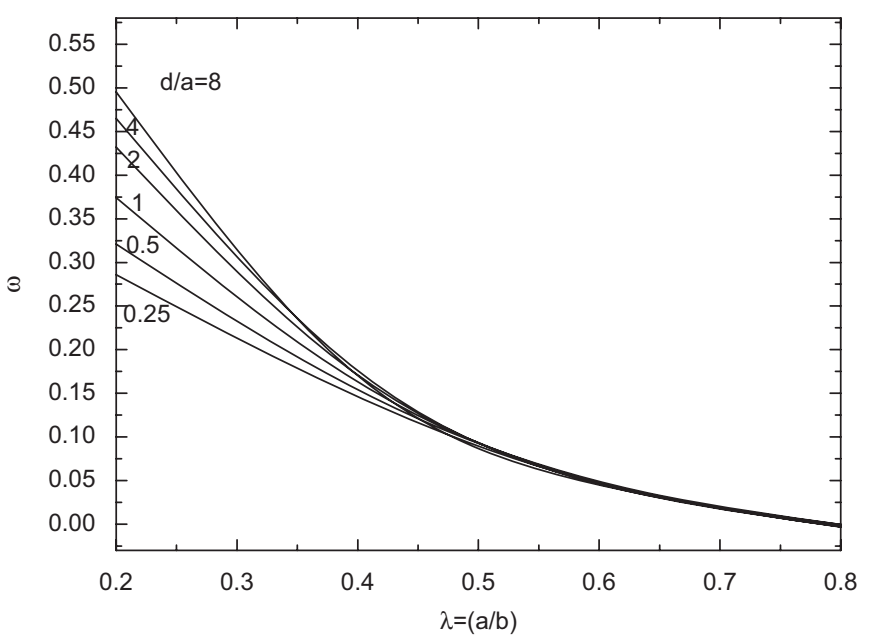

b

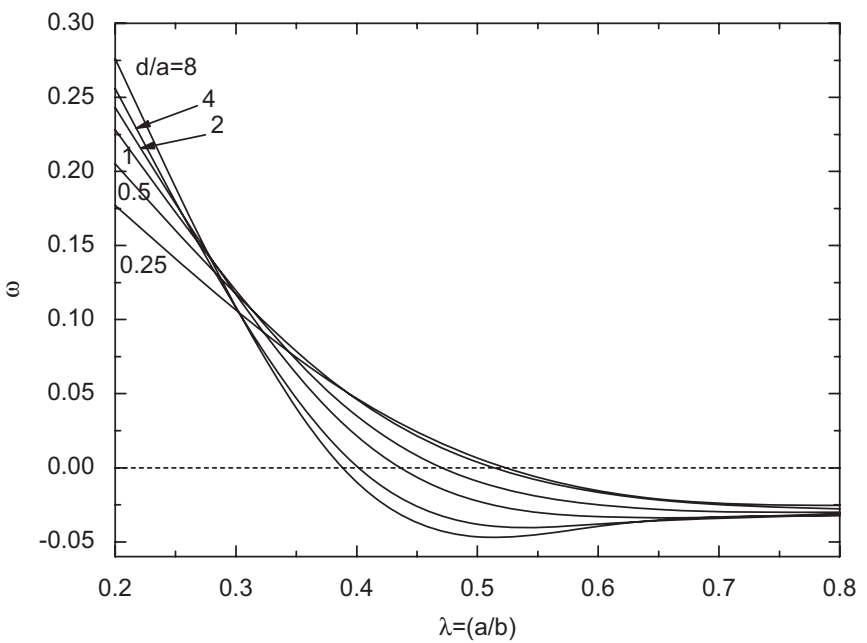

Fig. 10. Variation of the scaled electrophoretic mobility $\omega$ as a function of $\lambda(=a / b)$ for various combinations of $(d / a)$ and $\phi_{r}$ at $\zeta_{b}^{*}=0.2 \zeta_{a}^{*}$ and $\kappa a=1$. (a) $\phi_{r}=1$, (b) $\phi_{r}=5$.

to that for the case where the particle is positively charged and the pore is uncharged, except that the value of $\omega$ in the former is smaller than that in the latter. However, if $\phi_{r}$ is high, they are different both quantitatively and qualitatively. In particular, the $\omega$ in Fig. 10(b) can have a negative local minimum as $\lambda$ varies if the aspect ratio of the particle $(d / a)$ is sufficiently large. Again, this arises from the effects of double-layer polarization, the negative charge induced on the particle surface, and the electroosmotic flow.

\section{Conclusions}

The influences of the presence of a boundary, the effect of double-layer polarization, and that of electroosmotic flow on the electrophoretic behavior of a particle are discussed by considering the electrophoresis of a rigid, finite cylindrical particle along the axis of a long, charged cylindrical pore. The results of numerical simulation can be summarized as follows. (a) The presence of a positively charged pore leads to two effects that are absent in the case where it is uncharged, namely, negative charge is induced on the particle surface as it approaches the pore, and an electroosmotic flow is developed by the charged pore. Both of these effects will reduce the electrophoretic velocity of a positively charged particle. (b) For the case of a positively charged particle and an uncharged

pore if the surface potential of the particle is low and the thickness of the double layer takes a small to medium large value, the mobility of the particle increases monotonically with the decreasing double-layer thickness, but the reverse trend is observed for the case of a positively charged particle and a positively charged pore. On the other hand, if the surface potential of the particle is high, the mobility in the former case is always positive as the double-layer thickness declines, but that in the latter case may change its sign from positive to negative and from negative to positive again. These findings have practical significance because the change in the direction of electrophoresis will influence the prediction of the charged conditions on a particle if electrophoresis is used as an analytical tool, or the separation efficiency if it is adopted as a separation technique. (c) For a positively charged particle, if its surface potential is low, the behavior of its mobility for the case of a positively charged pore is similar to that of an uncharged pore, except that the mobility in the former is smaller than that in the latter. However, if the surface potential is high, they are different both quantitatively and qualitatively. In particular, the mobility in the former can have a negative local minimum as the degree of boundary effect varies.

\section{Notation}

$a$

$b$

$C D$

$d$

$D_{j}$

e

$\boldsymbol{e}_{z}$

E

$\mathbf{E}$

$E_{Z}$

$E_{Z}^{*}$

$F_{i}$

$F_{d i}$

$F_{e i}$

$F_{d i}^{*}$

$F_{e i}^{*}$

$g_{j}$

$g_{j}^{*}$

$I_{0}$

$k_{B}$

$L_{C}$

$L_{T}$

$n$

$n_{j}$

$n_{j}^{*}$

$n_{j 0}$

$p$

$p^{*}$

$P e_{j}$

$p_{\text {ref }}$

$S$

$S^{*}$

$t$

$T$ radius of the particle, $\mathrm{m}$

radius of the pore, $m$

$=n_{2}^{*}-n_{1}^{*}$

length of the particle, $m$

diffusivity of ionic species $j, \mathrm{~m}^{2} / \mathrm{s}$

elementary charge, $\mathrm{C}$

unit vector in the $z$-direction

strength of the applied electric field, $\mathrm{m}^{2} / \mathrm{V} / \mathrm{s}$

applied electric field, $\mathrm{V} / \mathrm{m}$

strength of the applied electric field in the $z$-direction, $\mathrm{m}^{2} / \mathrm{V} / \mathrm{s}$

scaled strength of the applied electric field in the $z$-direction

total force acting on the particle in sub-problem $i, \mathrm{~N} / \mathrm{m}$ $z$-component of the hydrodynamic force in subproblem $i, \mathrm{~N} / \mathrm{m}$

$z$-component of the electrical force in sub-problem $i$, $\mathrm{N} / \mathrm{m}$

scaled $z$-component of the hydrodynamic force in subproblem $i$

scaled $z$-component of the electrical force in subproblem $i$

perturbed potential associated with ionic species $j, \mathrm{~V}$ scaled perturbed potential

zero-order modified Bessel function of the first kind

Boltzmann constant, $\mathrm{J} / \mathrm{K}$

length of the particle, $\mathrm{m}$

length of the pore, $m$

magnitude of a unit normal vector

number concentration of ionic species $j, 1 / \mathrm{m}^{3}$

scaled number concentration of ionic species $j, 1 / \mathrm{m}^{3}$

bulk number concentration associated with ionic species $j, 1 / \mathrm{m}^{3}$

hydrodynamic pressure, Pa

scaled hydrodynamic pressure

Peclet number of ionic species $j$

reference pressure, $\mathrm{Pa}$

particle surface

scaled particle surface

unit tangential vector

absolute temperature, $\mathrm{K}$ 
U velocity of the particle, $\mathrm{m} / \mathrm{s}$

$U_{E} \quad=\left(\varepsilon_{c}^{2} / \eta a\right), \mathrm{m} / \mathrm{s}$

$v \quad$ speed of the particle in the $z$-direction, $\mathrm{m} / \mathrm{s}$

v liquid velocity, $\mathrm{m} / \mathrm{s}$

$\mathbf{v}^{*} \quad$ scaled liquid velocity

$z^{*} \quad z$-component of a unit normal vector

$z_{1} \quad$ valence of cations

$z_{2} \quad$ valence of anions

\section{Greek letters}

$\alpha \quad=-z_{2} / z_{1}$

$\beta \quad$ proportional constant

$\nabla \quad$ gradient operator, $1 / \mathrm{m}$

$\nabla^{*} \quad$ scaled gradient operator

$\nabla^{2} \quad$ Laplace operator, $1 / \mathrm{m}^{2}$

$\nabla^{* 2} \quad$ scaled Laplace operator

$\varepsilon \quad$ dielectric constant, $\mathrm{C}^{2} / \mathrm{N} / \mathrm{m}^{2}$

$\zeta \quad$ surface (zeta) potential, $\mathrm{V}$

$\zeta_{a} \quad$ surface potential of the particle, $\mathrm{V}$

$\zeta_{b} \quad$ surface potential of the pore, $\mathrm{V}$

$\eta \quad$ viscosity, $\mathrm{kg} / \mathrm{m} / \mathrm{s}^{2}$

$\kappa \quad$ reciprocal Debye length, $\mathrm{m}$

$\lambda \quad=a / b$

$\mu \quad$ mobility, $\mathrm{m}^{2} / \mathrm{V} / \mathrm{s}$

$\rho \quad$ space charge density, $\mathrm{C} / \mathrm{m}^{3}$

$\sigma^{H *} \quad$ scaled shear stress tensor

$\phi_{1} \quad$ equilibrium potential, $\mathrm{V}$

$\phi_{2} \quad$ perturbed potential, $\mathrm{V}$

$\phi_{r} \quad$ scaled surface potential of the particle

$\phi_{j}^{*} \quad$ scaled potential

$\chi \quad$ proportional constant

$\omega \quad$ scaled electrophoretic mobility

\section{Acknowledgment}

This work is supported by the National Science Council of the Republic of China.

\section{References}

Davison, S.M., Sharp, K.V., 2006. Boundary effects on the electrophoretic motion of cylindrical particles: concentrically and eccentrically-positioned particles in a capillary. Journal of Colloid and Interface Science 303, 288-297.
Dukhin, S.S., Semenikhin, N.M., 1970. Theory of double layer polarization and its effect on the electrokinetic and electrooptical phenomena and dielectric constants of dispersed systems. Kolloid Z 32, 360-368.

FlexPDE, 2003. version 2.22. PDE Solutions, USA

Happel, J., Brenner, H., 1983. Low-Reynolds Number Hydrodynamics. Nijhoff, Boston. pp. 113-114.

Helmholtz, H., 1879. Studien uber electrische grenschichten. Annalen der Physik und Chimie 7, 337-387.

Henry, D.C 1931. The cataphoresis of suspended particles. I: The equation of cataphoresis. Proceedings of the Royal Society of London Series A 133, 106-129.

Hsieh, T.H., Keh, H.J., 2007. Boundary effect on electrophoresis of a colloidal cylinder with a nonuniform zeta potential distribution. Journal of Colloid and Interface Science 315, 343-354.

Hsu, J.P., Cheng, Z.S., 2007. Electrophoresis of a sphere along the axis of a long cylindrical pore: effect of double-layer polarization and electroosmotic flow. Langmuir 23, 6198-6204.

Hsu, J.P., Kao, C.Y., 2002. Electrophoresis of a finite cylinder along the axis of a cylindrical pore. Journal of Physical Chemistry B 106, 10605-10609.

Hsu, J.P., Ku, M.H., 2005. Boundary effect on electrophoresis: finite cylinder in a cylindrical pore. Journal of Colloid and Interface Science 283, 592-600.

Hsu, J.P., Kuo, C.C., 2006. Electrophoresis of a finite cylinder positioned eccentrically along the axis of a long cylindrical pore. Journal of Physical Chemistry B 110 17607-17615.

Hsu, J.P., Yeh, L.H., 2006. Comparison of three methods for the evaluation of the electric force on a particle in electrophoresis. Journal of the Chinese Institute of Chemical Engineers 37, 601-607.

Hsu, J.P., Chen, Z.S., Ku, M.H., Yeh, L.H., 2007a. Effect of a charged boundary on electrophoresis: sphere in spherical cavity at arbitrary potential and doublelayer thickness. Journal of Colloid and Interface Science 314, 256-263.

Hsu, J.P., Yeh, L.H., Ku, M.H., 2007b. Evaluation of the electric force in electrophoresis Journal of Colloid and Interface Science 305, 324-329.

Lee, E., Chu, J.W., Hsu, J.P., 1998. Electrophoretic mobility of a sphere in a spherical cavity. Journal of Colloid and Interface Science 205, 65-76.

Liu, H., Bau, H.H., Hu, H.H., 2004. Electrophoresis of concentrically and eccentrically positioned cylindrical particles in a long tube. Langmuir 20, 2628-2639.

O'Brien, R.W., White, L.R., 1978. Electrophoretic mobility of a spherical colloidal particle. Journal of the Chemistry Society Faraday Transactions II 74, 1607-1627.

Ohshima, H., Healy, T.W., White, L.R., 1983. Approximate analytic expressions for the electrophoretic mobility of spherical colloidal particles and the conductivity of their dilute suspensions. Journal of the Chemistry Society Faraday Transactions II 79, 1613-1628.

Overbeek, J.Th.G., 1943. Theory of the relaxation effect in electrophoresis. Kolloide Beihefte 74, 287-364.

Von Smoluchowski, M.Z., 1918. Versuch einer mathematischen theorie der koagulation kinetic kolloider lösungen. Zeitschrift für Physikalische Chemie 92, 129-168.

Wiersema, P.H., Loeb, A.L., Overbeek, J.Th.G., 1966. Calculation of electrophoretic mobility of a spherical colloid particle. Journal of Colloid and Interface Science 22, 78-99.

Ye, C., Sinton, D., Erickson, D., Li, D., 2002. Electrophoretic motion of a circular cylindrical particle in a circular cylindrical microchannel. Langmuir 18, 9095-9101. 